\title{
Long-lived fermions at AL3X
}

\author{
Daniel Dercks, ${ }^{1, *}$ Herbert K. Dreiner, ${ }^{2, \dagger}$ Martin Hirsch, ${ }^{3, *}$ and Zeren Simon Wang ${ }^{2, \S}$ \\ ${ }^{1}$ Deutsches Elektronen-Synchrotron DESY, Notkestraße 85, 22607 Hamburg, Germany \\ ${ }^{2}$ Physikalisches Institut der Universität Bonn, Bethe Center for Theoretical Physics, \\ Nußallee 12, 53115 Bonn, Germany \\ ${ }^{3}$ AHEP Group, Instituto de Física Corpuscular-CSIC/Universitat de València Edificio de Institutos de Paterna, \\ Apartado 22085, E-46071 València, Spain
}

(Received 3 January 2019; published 15 March 2019)

\begin{abstract}
Recently Gligorov et al. [V. V. Gligorov et al., Phys. Rev. D 99, 015023 (2019)] proposed to build a cylindrical detector named AL3X close to the ALICE experiment at interaction point (IP) 2 of the LHC, aiming for discovery of long-lived particles (LLPs) during Run 5 of the HL-LHC. We investigate the potential sensitivity reach of this detector in the parameter space of different new-physics models with long-lived fermions namely heavy neutral leptons (HNLs) and light supersymmetric neutralinos, which have both not previously been studied in this context. Our results show that the AL3X reach can be complementary or superior to that of other proposed detectors such as CODEX-b, FASER, MATHUSLA and SHiP.
\end{abstract}

DOI: 10.1103/PhysRevD.99.055020

\section{INTRODUCTION}

There has recently been an increased interest in neutral long-lived particles (LLPs). They arise naturally in various models of dark matter or baryogenesis, for example. For reviews and further models see Refs. [1,2]. Surprisingly, even though not designed for this purpose, the LHC detectors ATLAS and CMS, have a relevant sensitivity to LLPs [3-6]. However, there are significant gaps in sensitivity to these models at the LHC. Thus several new experiments have recently been proposed, to specifically look for LLPs. These include the beam dump experiment SHiP [2] at the SPS at CERN. Further experiments are MATHUSLA [7], CODEX-b [8] and FASER [9], which would all be located at various positions with respect to the interaction points (IPs) of ATLAS or CMS, and would thus make use of LHC events. They are all shielded from the IPs by between $25 \mathrm{~m}$ and $450 \mathrm{~m}$ of rock. Very recently a further experiment has been proposed, AL3X [10], which would be located at the ALICE site at the LHC. It differs from MATHUSLA, CODEX-b and FASER in that the center of the detector is located only $11.25 \mathrm{~m}$ from the ALICE IP, and furthermore the detector would have a magnet. Due to

\footnotetext{
*daniel.dercks@desy.de

dreiner@uni-bonn.de

*mahirsch@ific.uv.es

${ }^{\S}$ wzeren@physik.uni-bonn.de
}

Published by the American Physical Society under the terms of the Creative Commons Attribution 4.0 International license. Further distribution of this work must maintain attribution to the author(s) and the published article's title, journal citation, and DOI. Funded by SCOAP. the proximity to the IP the experiment would have a significantly higher geometric acceptance, even for a comparatively small detector, than the other three proposed new experiments at the LHC. Such a small detector could be equipped with dense tracking instrumentation, which would be further improved by the magnetic field.

A subclass of interesting LLPs are heavy neutral fermions (HNFs). In this paper we shall focus on two examples: (a) heavy neutral leptons (HNLs). These are often also called sterile neutrinos in the literature. However we prefer the term HNL, because within the experimental neutrino oscillation community sterile neutrinos are usually identified with neutrinos with masses of order $\mathcal{O}(\mathrm{eV})$, whereas we shall focus on masses between 0.1 and $10 \mathrm{GeV}$. (b) The lightest neutralino in supersymmetry (SUSY), which can decay via R-parity violating (RPV) interactions. Somewhat surprisingly a light neutralino with a mass between 0.5 and $5 \mathrm{GeV}$, which we shall consider, is still consistent with all observations [11-14]. ${ }^{1}$ Recently we have investigated the search sensitivity of MATHUSLA, CODEX-b and FASER for these specific HNFs $[15,16]$.

It is the purpose of this paper to directly extend this recent work and investigate the sensitivity of the proposed AL3X detector to these HNFs, i.e., sterile neutrinos, as well as the lightest neutralino in supersymmetry, as they were not considered in the original AL3X paper [10]. For the HNLs we shall consider the production at the ALICE IP via $D$-and $B$-mesons. For the neutralinos we also consider the

\footnotetext{
${ }^{1}$ In fact even a massless neutralino is consistent with all observations [14].
} 
production via the decay of $D$ - and $B$-mesons, and in addition the direct pair production via $Z$-bosons.

The outline of the paper is as follows. In Sec. II we discuss the proposed AL3X detector set-up and define the parameters for our analysis. We furthermore present the details of our simulation of the long-lived HNFs. In Sec. III we present our results for the sensitivity of AL3X to longlived HNLs. In Sec. IV we present our results for the sensitivity of AL3X of long-lived light neutralinos. We consider separately the pair-production via $Z^{0}$ decays and the single production via rare heavy meson decays. In Sec. V we summarize and offer our conclusions.

\section{SIMULATION AND DETECTOR}

In this section we outline our simulation procedure and introduce the setup of the proposed detector AL3X. Throughout this work we assume zero background events and $100 \%$ detector efficiency. See the discussion in Ref. [10].

\section{A. Simulation procedure}

In order to obtain the expected number of detectable decay events, we estimate the total number, $N_{M}$, of mother particles $M$ produced at the LHC from existing experimental results. Here $M$ can be a $D$ - or a $B$-meson, or a $Z$-boson. We then calculate the branching ratio of the various $M \mathrm{~s}$ into the LLP(s), and compute the average decay probability of these LLPs inside the decay chamber of AL3X. We implement this aspect in a manner very similar to the treatment applied in our previous work, Refs. [15,16]. Note, that for $\operatorname{BR}\left(Z \rightarrow \tilde{\chi}_{1}^{0} \tilde{\chi}_{1}^{0}\right)$ we have only an experimental upper limit. We will assume two different values of $\operatorname{BR}\left(Z \rightarrow \tilde{\chi}_{1}^{0} \tilde{\chi}_{1}^{0}\right)$ in our numerical study for illustration.

Since the rare decays of charm and bottom mesons into HNLs lead to the strongest sensitivity reach in HNL mass $m_{N}$ and mixing square $\left|V_{\alpha N}\right|^{2}(\alpha=e, \mu)$, defined in Sec. III, we focus on these channels, discarding the complementary contributions from $W-, Z$ - and Higgs bosons. ${ }^{2}$ Similarly, in the case when an RPV $L Q \bar{D}$ coupling induces single production of a neutralino, we consider only rare decays of $D$ - and $B$-mesons, as well. From results published by the LHCb collaboration $[17,18]$, we estimate the number of produced mesons over a hemisphere for an integrated luminosity of $\mathcal{L}=100 / \mathrm{fb}$ :

$$
\begin{array}{lll}
N_{D^{ \pm}}=5.27 \times 10^{14}, & N_{D_{s}^{ \pm}}=1.70 \times 10^{14}, & N_{D_{0}}=1.00 \times 10^{15}, \\
N_{B^{ \pm}}=2.43 \times 10^{13}, & N_{B^{0}}=2.43 \times 10^{13}, & N_{B_{s}^{0}}=5.48 \times 10^{12}, \\
N_{B_{c}^{ \pm}}=5.54 \times 10^{10} .
\end{array}
$$

\footnotetext{
${ }^{2}$ The latter were, however, taken into account in Ref. [15].
}

Besides the LLPs produced from rare meson decays, we furthermore include the case of light neutralinos pairproduced from $Z$-boson decays. Experimentally viable light neutralinos must be dominantly binolike $[11,12]$, with only a small Higgsino component. It is the latter, which couples to the Z-boson. However, given the large cross section for $Z$-boson production at the LHC, we may still obtain good sensitivity reach in $L Q \bar{D}$ couplings up to a neutralino mass roughly half of the $Z$-boson mass. ATLAS published the experimentally measured cross section of $Z \rightarrow \ell^{+} \ell^{-}(\ell=e, \mu)$ in $p p$ collisions at $\sqrt{s}=13 \mathrm{TeV}$ [19]. With the $\operatorname{BR}\left(Z \rightarrow \ell^{+} \ell^{-}\right)$given by the PDG [20], we estimate the number of $Z$-bosons produced to be

$$
N_{Z}=2.94 \times 10^{9} \text {, }
$$

over a hemisphere for $\mathcal{L}=100 / \mathrm{fb}$.

We write the total number of LLPs produced, $N_{\mathrm{LLP}}^{\mathrm{prod}}$, as

$$
\begin{aligned}
N_{\mathrm{LLP}}^{\mathrm{prod}} & =\sum_{M} N_{M} \cdot \Gamma(M \rightarrow \operatorname{LLP}(\mathrm{s})+X) \cdot \tau_{M} \\
& =\sum_{M} N_{M} \cdot \operatorname{BR}(M \rightarrow \operatorname{LLP}(\mathrm{s})+X),
\end{aligned}
$$

where $M$ can be either a $D$ - or a $B$-meson, or a $Z$-boson, and with $\tau_{M}$ denoting its lifetime. To determine the average decay probability of the LLPs inside the AL3X "detectable region"(“d.r."), $\langle P[$ LLP in d.r. $]\rangle$, we perform a Monte Carlo (MC) simulation with PYTHIA 8.205 [21,22]. We implement the following formula:

$$
\langle P[\text { LLP in d.r. }]\rangle=\frac{1}{N_{\mathrm{LLP}}^{\mathrm{MC}}} \sum_{i=1}^{N_{\mathrm{LLP}}^{\mathrm{MC}}} P\left[(\mathrm{LLP})_{i} \text { in d.r. }\right],
$$

where $N_{\mathrm{LLP}}^{\mathrm{MC}}$ is the number of LLPs generated in the MC simulation sample. We generate the $D$ - and $B$-mesons by making use of the matrix element calculators HardQCD : hardccbar and HardQCD: hardbbbar, respectively, of PythiA. In order to extract the kinematics of pairproduced neutralinos from $Z$-boson decays, we resort to the "new-gauge-boson processes" provided by PYтнIA to generate pure $Z^{\prime}$-bosons with the same mass as the Standard Model (SM) Z-boson and let it decay to a pair of new fermion particles. Finally, we calculate the number of observed decays of the LLPs in the detector,

$N_{\mathrm{LLP}}^{\mathrm{obs}}=N_{\mathrm{LLP}}^{\mathrm{prod}} \cdot\langle P[$ LLP in d.r. $]\rangle \cdot \mathrm{BR}(\mathrm{LLP} \rightarrow$ visible only $)$,

where we also include $\mathrm{BR}(\mathrm{LLP} \rightarrow$ visible only), the branching ratio of the LLP into only visible states such that the event may be reconstructed by AL3X. In Ref. [10] it was pointed out that the LLP vertex is required, in order 
to point back to the IP, and thus be able to reduce the background.

With PYTHIA providing the kinematical information of each generated LLP, we can easily derive its velocity $\beta_{i}$ and Lorentz boost factor $\gamma_{i}$. We calculate the total decay width of the HNLs by using the formulas given in Ref. [23]. As for the decay width of the light neutralinos, we use the relevant expressions for neutralino two-body decays given in Ref. [6] for a neutralino mass below $\sim 3.5 \mathrm{GeV}$, and take the three-body decay results given by SPHENO 4.0.3 [24,25] for larger masses. Combining the total decay width $\Gamma_{\text {tot }}($ LLP $)$ with the $\beta_{i}$ and $\gamma_{i}$, we express the decay length, $\lambda_{i}$, of a given LLP, (LLP) $)_{i}$, in the laboratory frame:

$$
\begin{aligned}
& \lambda_{i}=\beta_{i} \gamma_{i} / \Gamma_{\text {tot }}(\text { LLP }), \\
& \lambda_{i}^{z}=\beta_{i}^{z} \gamma_{i} / \Gamma_{\text {tot }}(\text { LLP }),
\end{aligned}
$$

where $\lambda_{i}^{z}$ is the $z$-component of $\lambda_{i}$ along the beam axis. The decay length is required in order to calculate the decay probability $P\left[(\mathrm{LLP})_{i}\right.$ in d.r.].

\section{B. The AL3X detector}

AL3X [10] is proposed as an on-axis cylindrical detector situated several meters from IP2 in the ALICE/L3 cavern at the LHC. It has a length of $L_{d}=12 \mathrm{~m}$ and an inner/outer radius of $0.85 / 5 \mathrm{~m}$. In virtue of its proximity to the IP, its pseudorapidity coverage of $[0.9,3.7]$ is large relative to other proposed future detectors such as MATHUSLA $([0.9,1.8])[7]$ and CODEX-b $([0.2,0.6])[8]$, and it has a full azimuthal coverage. ${ }^{3}$ We calculate the probability of each individual LLP decaying inside the detector chamber $P\left[(\mathrm{LLP})_{i}\right.$ in d.r. $]$ as:

$$
\begin{aligned}
& P\left[(\text { LLP })_{i} \text { in d.r. }\right]=e^{-\frac{L_{i}}{\lambda_{i}^{2}}}\left(1-e^{-\frac{L_{i}^{\prime}}{\lambda_{i}^{2}}}\right), \\
& L_{i}=\min \left(\max \left(L_{h}, \frac{L_{v}}{\tan \theta_{i}}\right), L_{h}+L_{d}\right), \\
& L_{i}^{\prime}=\min \left(\max \left(L_{h}, \frac{L_{v}+H}{\tan \theta_{i}}\right), L_{h}+L_{d}\right)-L_{i},
\end{aligned}
$$

where $L_{h}=5.25 \mathrm{~m}$ is the horizontal distance from the IP to the near end of the detector, $L_{v}=0.85 \mathrm{~m}$ and $H=4.15 \mathrm{~m}$ are respectively the inner radius and the transverse length of the detector, and $\theta_{i}$ is the polar angle of (LLP) $)_{i}$ with respect to the beam axis. In Ref. [10] the authors employed the benchmark integrated luminosities $100 / \mathrm{fb}$ and $250 / \mathrm{fb}$, so that practical concerns such as moving the IP and beam quality, and constraints from backgrounds may be

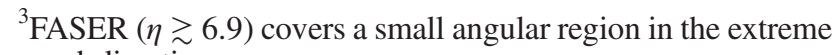
forward direction.

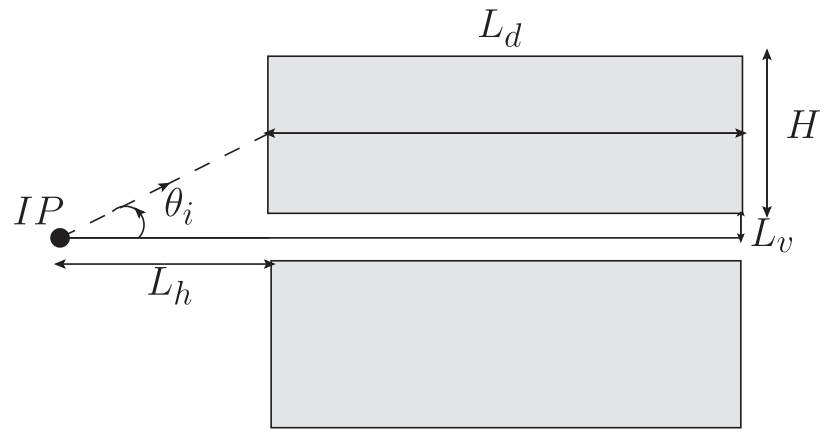

FIG. 1. Side-view sketch of the AL3X detector with definition of distances and angles used in text. The detector is cylindrically symmetric around the beam axis. IP denotes the interaction point 2 at the LHC. The dashed line describes an example LLP track, with polar angle $\theta_{i}$.

investigated. Here we follow their choice of luminosities. In Fig. 1 we show a profile sketch of AL3X.

Before we present the sensitivity estimates, we compare the average decay probability $\langle P[$ LLP in d.r. $]\rangle$, also called fiducial efficiencies $\epsilon_{\text {fid }}$ in Ref. [10], of AL3X and MATHUSLA for the different benchmark models considered in this study. In order to present our results for $\epsilon_{\text {fid }}$ with a linear dependence on $c \tau$, we estimate $\epsilon_{\text {fid }}$ in the limit that the decay length $\beta \gamma c \tau$ is much larger than the distance from the IP to the detector. In the calculation we use the exact formula. We take the benchmark LLP mass as $1 \mathrm{GeV}$ for both HNLs and light neutralinos. For LLPs of $1 \mathrm{GeV}$ produced from charm and bottom meson decays, the typical $\beta \gamma$ value is of order $\mathcal{O}(1)$, see Table II in Ref. [16]. Thus we require to evaluate $\epsilon_{\text {fid }}$ at $c \tau=100 \mathrm{~m}$, and our results in Table I are roughly valid only for $c \tau \geq 100 \mathrm{~m}$. On the other hand, LLPs from $Z$-boson decays have a $\beta \gamma$ of order $\mathcal{O}(100)$, so our results of $\epsilon_{\text {fid }}$ in this case are valid roughly for $c \tau \geq 1 \mathrm{~m}$. The results are shown in Table $\mathrm{I},{ }^{4}$ where $\epsilon_{\text {fid }}^{\mathrm{HNL}-\mathrm{D}}, \epsilon_{\mathrm{fid}}^{\mathrm{HNL}-\mathrm{B}}, \epsilon_{\mathrm{fid}}^{\tilde{\chi}_{1}^{0}-D_{s}^{+}}, \epsilon_{\text {fid }}^{\tilde{\chi}_{1}^{0}-B_{0}}$ and $\epsilon_{\text {fid }}^{\tilde{\chi}_{1}^{0}-Z}$ denote respectively the fiducial efficiencies for HNLs produced from $D$ - and $B$-meson decays, light neutralinos produced from $D_{s}^{+}$and $B_{0}$ decays, and light neutralinos pair-produced from $Z$-boson decays. In general one finds that in the large decay length regime AL3X has slightly larger fiducial efficiencies than MATHUSLA in these benchmark scenarios.

\section{HEAVY NEUTRAL LEPTONS}

In this section we discuss the prospects of AL3X for detecting heavy neutral leptons HNLs. HNLs, $N_{j}$, have charged (CC) and neutral current (NC) interactions,

\footnotetext{
${ }^{4}$ Our estimates of the fiducial efficiencies for the lightest neutralinos pair-produced from $Z$-boson decays are somewhat smaller than those given in Ref. [10], where Higgs decays were considered. This is because Ref. [10] applies the approximation that $\beta \gamma c \tau$ is much larger than the distance from the IP to the detector in the calculation, while we use the exact expression.
} 
TABLE I. Summary of fiducial efficiencies of AL3X and MATHUSLA for different models with the benchmark LLP mass at 1 GeV.

\begin{tabular}{|c|c|c|c|c|c|}
\hline Detector & $\epsilon_{\mathrm{fid}}^{\mathrm{HNL}-\mathrm{D}} \cdot c \tau / m$ & $\epsilon_{\mathrm{fid}}^{\mathrm{HNL}-\mathrm{B}} \cdot c \tau / m$ & $\epsilon_{\mathrm{fid}}^{\tilde{x}_{1}^{0}-D_{s}^{+}} \cdot c \tau / m$ & $\epsilon_{\mathrm{fid}}^{\tilde{\chi}_{1}^{0}-B_{0}} \cdot c \tau / m$ & $\epsilon_{\text {fid }}^{\tilde{x}_{1}^{0}-Z} \cdot c \tau / m$ \\
\hline AL3X & $4.8 \times 10^{-1}$ & $4.6 \times 10^{-1}$ & $3.9 \times 10^{-1}$ & $2.3 \times 10^{-1}$ & $1.6 \times 10^{-2}$ \\
\hline MATHUSLA & $9.7 \times 10^{-2}$ & $1.3 \times 10^{-1}$ & $1.1 \times 10^{-1}$ & $1.2 \times 10^{-1}$ & $8.0 \times 10^{-4}$ \\
\hline
\end{tabular}

suppressed relative to electroweak strength via small mixing elements:

$$
\begin{aligned}
\mathcal{L}= & \frac{g}{\sqrt{2}} V_{\alpha N_{j}} \bar{\ell}_{\alpha} \gamma^{\mu} P_{L} N_{j} W_{L \mu}^{-} \\
& +\frac{g}{2 \cos \theta_{W}} \sum_{\alpha, i, j} V_{\alpha i}^{L} V_{\alpha N_{j}}^{*} \bar{N}_{j} \gamma^{\mu} P_{L} \nu_{i} Z_{\mu},
\end{aligned}
$$

where $i=1,2,3$ and $j=1, \ldots, n$, and $\ell_{\alpha}, \alpha=e, \mu$, are the charged leptons of the SM. For kinematic reasons, we restrict ourselves to the first two generations. $V_{\alpha N_{j}}$ denotes the mixing between ordinary neutrinos and the HNLs of mass $m_{N_{j}}$. The mixing $\left|V_{\alpha N_{j}}\right|$ controls both production and decay of the HNLs.

HNLs/sterile neutrinos are mostly motivated by their connection with the generation of masses for the light, active neutrinos. In the standard minimal seesaw picture one simply adds three fermionic singlets to the SM, together with their Majorana mass terms. Within this simplest model, one expects that these steriles mix with the active neutrinos roughly at the order of $V_{\alpha N_{j}} \propto \sqrt{m_{\nu} / m_{N}}$, i.e., $\left|V_{\alpha N_{j}}\right|^{2} \simeq 5 \times 10^{-11}\left(\frac{m_{\nu}}{0.05 \mathrm{eV}}\right)\left(\frac{1 \mathrm{GeV}}{m_{N}}\right)$. However, other model variants, such as the inverse seesaw [26], lead to much larger mixing, despite the smallness of the observed neutrino masses. Below, we take $\left|V_{\alpha N_{j}}\right|^{2}$ as a free parameter in our calculations. ${ }^{5}$

We now turn to the discussion of the results. Figure 2 shows sensitivity estimates for AL3X and various other recent experimental proposals to HNLs. For AL3X we show two curves, one for $100 / \mathrm{fb}$ and one for $250 / \mathrm{fb}$, corresponding to the two options discussed in Ref. [10]. The grey area in the background shows the parameter space currently excluded according to Ref. [28] by the searches from PS191 [29], JINR [30], CHARM [31], and DELPHI [32]. Sensitivities for HNLs for CODEX-b (300/fb) [8], FASER (3/ab) [9] and MATHUSLA (3/ab) [33] have been calculated in Ref. [15]. While we use Ref. [15] in this plot, we note that these estimates agree quite well with other calculations for the same experiments in Ref. [7] (for MATHUSLA) and Ref. [34] (FASER). The line for LBNE is taken from Ref. [35], SHiP $\left(2 \times 10^{20}\right.$ protons on target $)$ from Ref. [36], while the final sensitivity of NA62 was recently estimated in Ref. [37].

\footnotetext{
${ }^{5}$ See also Ref. [27] for a detailed computation of the seesaw model.
}

Figure 2 shows that AL3X is quite competitive for the search of HNLs, with a sensitivity better than FASER, CODEX-b or NA62, even for only $100 / \mathrm{fb}$ of statistics. In the mass range above $m_{N} \sim 2 \mathrm{GeV}, \mathrm{AL} 3 \mathrm{X}$ has a sensitivity that is better than the estimate for SHiP [36], and only slightly worse than MATHUSLA. Below $m_{N} \sim 2 \mathrm{GeV}$, SHiP gives the best sensitivity, with AL3X@250/fb only roughly a factor (2-3) less sensitive than MATHUSLA in that mass range. Note, however, that the estimate for MATHUSLA is based on $3 / \mathrm{ab}$ of statistics.

\section{LIGHT NEUTRALINOS DECAYING VIA R-PARITY VIOLATION}

We continue with a discussion of the expected sensitivity of AL3X to a light long-lived neutralino in RPV SUSY [38,39]. Supersymmetric theories are an interesting extension to the SM $[40,41]$. In supersymmetry, the fermionic partners of the neutral gauge bosons and the neutral $C P$ even scalar Higgs fields mix to form four mass eigenstates called neutralinos, and denoted $\tilde{\chi}_{i}^{0}$. The lightest of these, $\tilde{\chi}_{1}^{0}$, is typically the lightest particle of the supersymmetric spectrum (LSP).

Rules of constructing gauge-, Lorentz- and SUSYinvariant Lagrangians reproduce the known interactions of the SM, and however additionally predict the following operators in the superpotential [42]

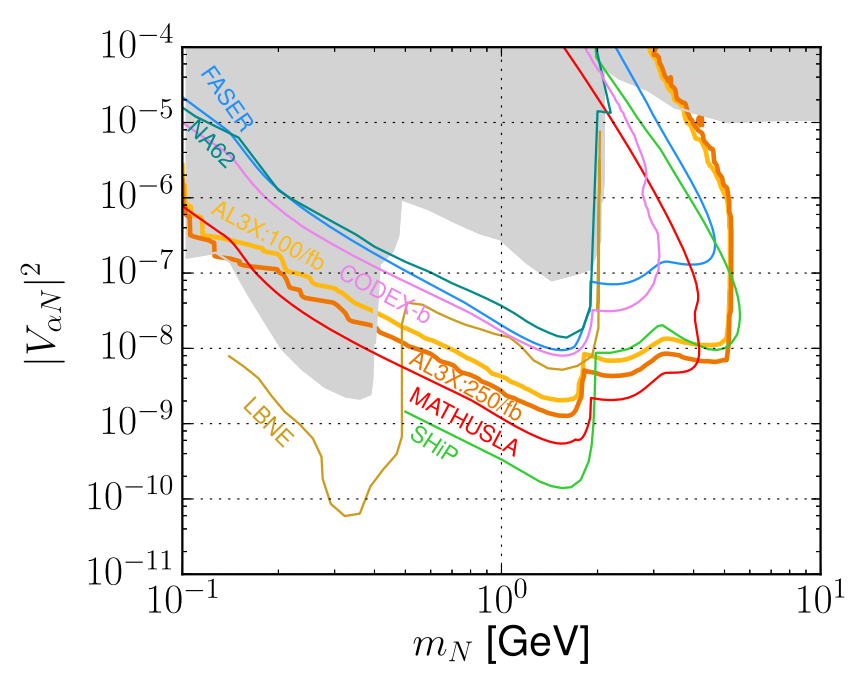

FIG. 2. Estimates for the sensitivity of different experiments to HNLs in the plane mixing angle squared, $\left|V_{\alpha N}\right|^{2}$, versus mass of the HNL, $m_{N}[\mathrm{GeV}]$. The references for the individual curves are given in the text. 


$$
\begin{aligned}
W_{\mathrm{RPV}}= & \kappa_{i} L_{i} H_{u}+\lambda_{i j k} L_{i} L_{j} E_{k}^{c} \\
& +\lambda_{i j k}^{\prime} L_{i} Q_{j} D_{k}^{c}+\lambda_{i j k}^{\prime \prime} U_{i}^{c} D_{j}^{c} D_{k}^{c} .
\end{aligned}
$$

The presence of the dimensionful parameters $\kappa_{i}$, and/or the Yukawa couplings $\lambda_{i j k}$ and/or $\lambda_{i j k}^{\prime}$ leads to lepton-number violation, whilst a nonvanishing $\lambda_{i j k}^{\prime \prime}$ violates baryon number. In our study we only discuss the phenomenology of nonvanishing $\lambda^{\prime}$. This choice conserves baryon number and hence does not lead to unobserved decays of the proton. See also Refs. [43-48] on the motivation for this choice of couplings and on the changes in phenomenology due to RPV. The $L Q \bar{D}$ operators predict, among others, the following effective operators between the neutralino, and the SM fermions $u, d, \ell$ and $\nu$ :

$$
\begin{aligned}
\mathcal{L} \supset & G_{i a b}^{S, \nu}\left(\overline{\tilde{\chi}^{0}} P_{L} \nu_{i}\right)\left(\overline{d_{b}} P_{L} d_{a}\right) \\
& +G_{i a b}^{S, \ell}\left(\overline{\tilde{\chi}^{0}} P_{L} \ell_{i}\right)\left(\overline{d_{b}} P_{L} u_{a}\right)+\text { H.c. }
\end{aligned}
$$

The effective couplings $G$ depend on several masses of the scalar supersymmetric partners $\tilde{f}$ of the SM fermions, the mixing within the neutralino sector and are linear in $\lambda^{\prime}$. The exact formulas can be found in Ref. [6]. If all $\tilde{f}$ are mass degenerate $G$ can be written as $\mathcal{O}(1) \times \lambda_{\text {iab }}^{\prime} / m_{\tilde{f}}^{2}$. Bounds on various combinations of $\lambda_{i a b}^{\prime}$ and $m_{\tilde{f}}$ can be set from searches for exotic decays in the meson sector, see e.g., Ref. [49-52]. In the special case of mass degenerate $\tilde{f}$ they can be compared to our sensitivity curves as we show below.

The absence of any R-parity violating terms predicts a stable $\tilde{\chi}_{0}$. In contrast, the terms in Eq. (13) directly imply a long-lived particle, which eventually decays into SM fermions.

\section{A. Pair production of $\tilde{\chi}_{1}^{0}$ from $Z$-boson decays}

There are various possibilities to produce neutralinos at the LHC. One of these is the decay of on-shell $Z$ bosons into pairs of neutralinos if $m_{\tilde{\chi}_{1}^{0}} \lesssim m_{Z} / 2$. The corresponding partial decay width $\Gamma\left(Z \rightarrow \tilde{\chi}_{1}^{0} \tilde{\chi}_{1}^{0}\right)$ has been calculated in Ref. [53] and is proportional to (cf. Eq. (K.2.5) in Ref. [27])

$$
\left(\left|N_{13}\right|^{2}-\left|N_{14}\right|^{2}\right)^{2},
$$

where $N_{13}, N_{14}$ are the two neutral $C P$-even Higgsino admixtures of the lightest neutralino. A light neutralino is dominantly bino but can have a substantial Higgsino admixture $[11,14,15]$. However, even in that case, we see from Eq. (14), that there can be a cancellation leading to a vanishing $Z \rightarrow \tilde{\chi}_{1}^{0} \tilde{\chi}_{1}^{0}$ branching ratio. Therefore, the invisible $Z$ decay width can in principle be arbitrarily small. Rather than scan over the supersymmetric parameter space, we use $\operatorname{BR}\left(Z \rightarrow \tilde{\chi}_{1}^{0} \tilde{\chi}_{1}^{0}\right)$ as a free parameter, see e.g., Ref. [53] for the detailed dependence.
As discussed in the previous work of Refs. [14,15], typical values of the invisible branching ratio in supersymmetry is around $6 \times 10^{-4}$, while the experimental upper bounds on the invisible $Z$ branching ratio require values below $0.1 \%$ at $90 \% \mathrm{CL}$, according to Ref. [54]. For our analysis we therefore choose $\operatorname{BR}\left(Z \rightarrow 2 \tilde{\chi}_{1}^{0}\right)=10^{-3}$ and $10^{-5}, 6$ as two representative and experimentally viable values for this invisible branching ratio.

For this benchmark analysis, we choose $\lambda_{112}^{\prime}$ to be the only nonvanishing RPV operator. In that case, the neutralino can decay into $u+\bar{s}+e^{-}$and $d+\bar{s}+\nu_{e}$ final states, as well as their respective charge conjugates. We use these inclusive final states to calculate the total lifetime of the neutralino, see Ref. [15]. However, in practice it may only be feasible to detect charged final states with light mesons, $\tilde{\chi}_{1}^{0} \rightarrow K^{ \pm} e^{\mp}$. We also calculate the partial decay width into this particular final state according to Ref. [6] and multiply with the corresponding branching ratio. We determine results for both cases, i.e., if only the charged meson final state or if all hadronic final states can be observed.

Results for this scenario are shown in Fig. 3 for both benchmark values of the $\operatorname{BR}\left(Z \rightarrow 2 \tilde{\chi}_{1}^{0}\right)$. We choose the mass of the neutralino as one free parameter, which for kinematic reasons must be smaller than $m_{Z} / 2$. As explained above, RPV-induced decays of the neutralino depend on the effective coupling $\lambda^{\prime} / m_{\tilde{f}}^{2}$, which is why we choose this as our second free parameter. Current limits on the RPV operators $L_{1} Q_{1} \bar{D}_{2}$ are taken from Ref. [50] and compared to our results. Note, however, that such a comparison is only valid if all sfermions are mass degenerate. See the discussion in Ref. [6].

We observe that for invisible branching ratios close to the current PDG limit, AL3X is sensitive to values of $\lambda_{112}^{\prime} / m_{\tilde{f}}^{2}$ down to $10^{-12} \mathrm{GeV}^{-2}$, if all hadronic final states can be observed. This strongest sensitivity is reached for neutralino masses near the kinematic threshold, at $m_{\tilde{\chi}_{1}^{0}} \approx 40 \mathrm{GeV}$. The sensitivity drops by nearly one order of magnitude, if only the charged final state $K^{ \pm} e^{\mp}$ is taken into account. Note that this search is more sensitive than current limits on the $\lambda_{112}^{\prime}$ coupling by several orders of magnitude. The lighter the neutralino, the lower the sensitivity on $\lambda_{112}^{\prime} / m_{\tilde{f}}^{2}$ but even for $\mathcal{O}(\mathrm{GeV})$ masses AL3X may be expected to significantly improve on current limits. Note that smaller neutralino masses have a reduced accessible final state phase space and hence a reduced difference in sensitivity between the conservative " $K^{ \pm} e^{\mp "}$ " and the optimistic "all hadronic final states."

\footnotetext{
${ }^{6}$ Here and elsewhere the values chosen for $\operatorname{BR}\left(Z \rightarrow \tilde{\chi}_{1}^{0} \tilde{\chi}_{1}^{0}\right)$ should be understood as the branching ratio of $Z$ boson to a pair of $\tilde{\chi}_{1}^{0}$ 's for $m_{\tilde{\chi}_{1}^{0}} \ll m_{Z}$. With $m_{\tilde{\chi}_{1}^{0}}$ increased up to the threshold $m_{Z} / 2$, phase space suppression effect becomes important and is correctly taken into account in our study.
} 


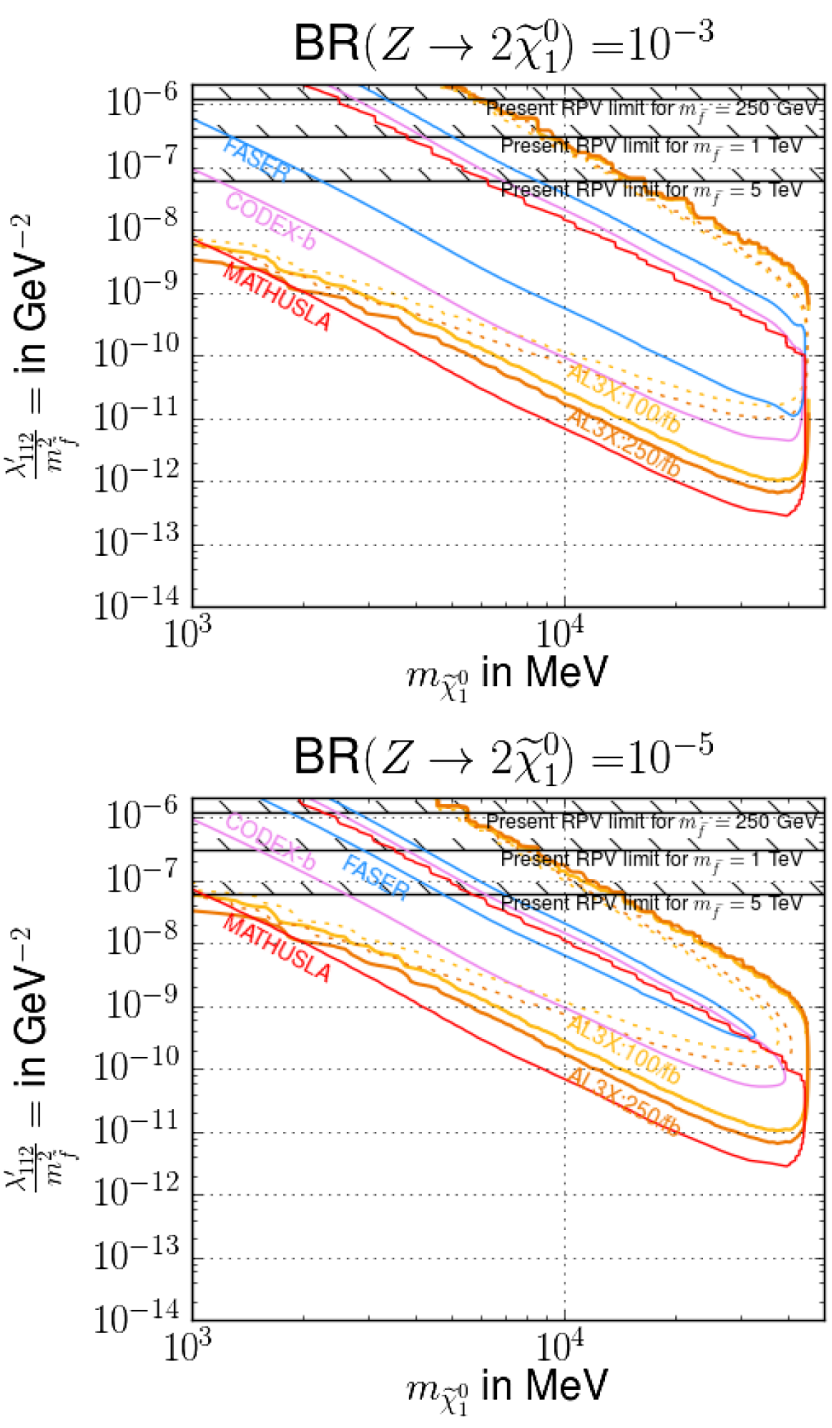

FIG. 3. AL3X sensitivity shown in the plane spanned by the neutralino mass and the effective RPV coupling $\lambda_{112}^{\prime} / m_{\tilde{f}}^{2}$ for two different assumptions of the $Z$ branching ratio to neutralinos. Sensitivity curves denote the expected measurement of 3 visible events with an integrated luminosity of $100 / \mathrm{fb}$ and $250 / \mathrm{fb}$. Also shown for comparison are the sensitivities of other experiments, taken from Ref. [15]. Solid lines consider all hadronic final states while dashed lines - only evaluated for AL3X in this work-only consider the branching ratio into the charged $K^{ \pm} e^{\mp}$ final state for observable neutralino decays. Overlaid current RPV limits on $\lambda_{112}^{\prime}$ are shown for comparison, using different assumptions on the degenerate sfermion mass $m_{\tilde{f}}$. The references are given in the text.

In comparison to the other proposed experiments, AL3X outperforms FASER and CODEX-b over the entire parameter range. It is competitive with MATHUSLA at the lowvalue range of $\lambda_{112}^{\prime}$ and neutralino masses of a few GeV. For larger masses, MATHUSLA shows the strongest sensitivity for small RPV operators.

Note that too large values of $\lambda^{\prime}$ render the neutralino too short-lived to reach the detector which leads to upper bounds on the sensitivity to $\lambda^{\prime}$ for all LLP experiments. In comparison to FASER, MATHUSLA and CODEX-b, AL3X covers the largest region of parameter space here, due to its proximity to IP2. However, for masses above $10 \mathrm{GeV}$, sizable RPV couplings may still evade both current detection limits and even the limits from AL3X@250/fb.

\section{B. Single production of $\tilde{\chi}_{1}^{0}$ from rare $D$ - and $B$-meson decays}

The effective operators in Eq. (13) mediate interactions between the neutralino, the SM leptons and SM mesons. Depending on the masses, a single operator can either predict the decay of a SM meson $M$ into $\tilde{\chi}_{1}^{0}+\ell$ or predict the decay of a neutralino into $\ell+M$ with small width and hence long lifetime. Here $\ell$ denotes either a charged or neutral lepton. In this study we assume that in any given model there are two nonvanishing couplings: $\lambda_{\text {prod }}^{\prime}, \lambda_{\text {dec }}^{\prime}$, at a time. These are respectively responsible for the production of neutralinos via the decay of a heavier meson, and for the decay of neutralinos into a lighter mesons. For simplicity, we here only consider low-energy models and do not take the effect of generating additional couplings via renormalization group equations into account [55-57].

In this study, we exemplarily choose two benchmark scenarios with different choices for the nonvanishing $\lambda^{\prime}$, summarized in Table II. We choose these two scenarios because they are representative for a class of $L Q \bar{D}$ couplings combinations. For more details, see the discussion below. For each scenario we have a different initial meson flavor, which produces the neutralinos via decays. This is important as these differ in their LHC production yields, see Eq. (1), as well as the different final states the neutralinos decay into. Due to the simultaneous presence of several operators from one $\lambda^{\prime}$ coupling, see Eq. (13), we often expect both charged and neutral final states. We call the former "visible," as only those can be experimentally measured by an LLP experiment. We need to consider all possible final states for the total lifetime of the lightest neutralino, $\tau_{\tilde{\chi}}$, but multiply the final number with the "visible branching ratio," i.e., the fraction of decays into a charged final state. More details on these benchmarks,

TABLE II. Features of the R-parity violating benchmark scenarios studied in this section.

\begin{tabular}{lcc}
\hline \hline & Scenario 1 & Scenario 2 \\
\hline$\lambda_{\text {prod for production }}^{\prime}$ & $\lambda_{122}^{\prime}$ & $\lambda_{131}^{\prime}$ \\
$\lambda_{\text {dec }}^{\prime}$ for decay & $\lambda_{112}^{\prime}$ & $\lambda_{112}^{\prime}$ \\
Produced meson(s) & $D_{s}$ & $B^{0}, \bar{B}^{0}$ \\
Visible final state(s) & $K^{ \pm} e^{\mp}, K^{* \pm} e^{\mp}$ & $K^{ \pm} e^{\mp}, K^{* \pm} e^{\mp}$ \\
Invisible final state(s) & $\left(\eta, \eta^{\prime}, \phi\right)+\left(\nu_{e}, \bar{\nu}_{e}\right)$ & None \\
$\quad$ via $\lambda_{\text {prod }}^{\prime}$ & & \\
Invisible final & $\left(K_{L}^{0}, K_{S}^{0}, K^{*}\right)+\left(\nu_{e}, \bar{\nu}_{e}\right)$ & $\left(K_{L}^{0}, K_{S}^{0}, K^{*}\right)+(\nu, \bar{\nu})$ \\
$\quad$ state(s) via $\lambda_{\text {dec }}^{\prime}$ & & \\
\hline \hline
\end{tabular}


including formulas for the respective decay widths and branching ratios can be found in Ref. [6].

In Fig. 4 we show model-dependent sensitivity curves of the AL3X detector with an integrated luminosity of $250 / \mathrm{fb}$. As explained above, the responsible effective operators scale with $\lambda^{\prime} / m_{\tilde{f}}^{2}$, which is why we choose this ratio of parameters for our figure axes. Since the mass of the long-lived neutralino affects the final state kinematics and the accessible phase space, we choose three representative mass values for each scenario: $m_{\tilde{\chi}_{1}^{0}}=600,1200$ and $1800 \mathrm{MeV}$ for scenario 1 and 1000, 3000 and $5000 \mathrm{MeV}$ for scenario 2. Due to the required decay chain, scenario 1 is restricted to the mass range $m_{K} \leq m_{\tilde{\chi}_{1}^{0}} \leq m_{D_{s}}$ while
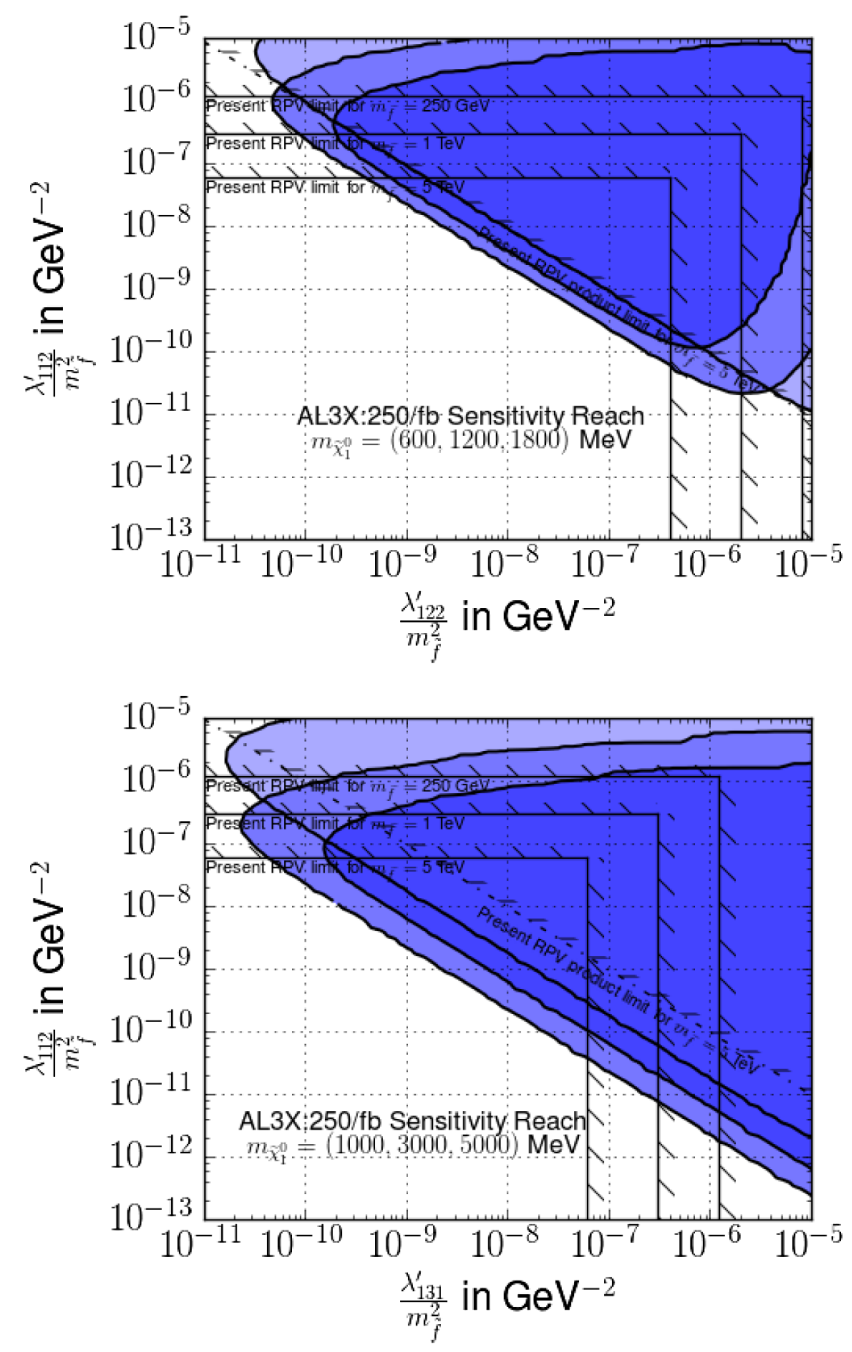

FIG. 4. AL3X sensitivity shown in the plane spanned by the two free parameters $\lambda_{\text {prod }}^{\prime}$ and $\lambda_{\mathrm{dec}}^{\prime}$ as respectively defined in Table II. Sensitivity curves denote the expected measurement of 3 visible events with an integrated luminosity of $250 / \mathrm{fb}$ for three different choices for the neutralino mass. The first/second/third value denoted in the plot respectively corresponds to the light blue/medium blue/dark blue region, respectively. Overlaid RPVlimits are shown for different choices of the sfermion mass $m_{\tilde{f}}$. scenario 2 requires $m_{K} \leq m_{\tilde{\chi}_{1}^{0}} \leq m_{B}$. For each figure, we also show the corresponding existing bound on the respective RPV operator taken from Ref. [50]. As mentioned, these can only be compared in the special case of mass degenerate sfermions.

For scenario 1 (2), AL3X is sensitive to values of $\lambda_{\text {prod }}^{\prime}$ down to $\lambda_{122}^{\prime} / m_{\tilde{f}}^{2}\left(\lambda_{131}^{\prime} / m_{\tilde{f}}^{2}\right)=3 \times 10^{-11}\left(2 \times 10^{-11}\right) \mathrm{GeV}^{-2}$, in case of relatively light neutralino masses, i.e., close to the lower mass threshold. Heavier masses can weaken this bound by up to an order of magnitude. The general bounds in the parameter planes then depend on the combination of both $\lambda_{\text {prod }}^{\prime}$ and $\lambda_{\text {dec }}^{\prime}$ since both couplings can simultaneously affect the neutralino lifetime and its visible branching ratio.

Note that for scenario 1, there exist both upper and lower bounds on $\lambda_{\mathrm{dec}}^{\prime} / m_{\tilde{f}}^{2}=\lambda_{112}^{\prime} / m_{\tilde{f}}^{2}$ whose precise values depend on the neutralino mass. If this coupling is chosen too large, the neutralino lifetime is too short and they decay before reaching AL3X. If it is too small, however, many neutralinos will live too long and hence one requires a large value for $\lambda_{\text {prod }}^{\prime}$ to produce enough neutralinos to still predict three observed decays. However, too large values of $\lambda_{\text {prod }}^{\prime} / m_{\tilde{f}}^{2}=\lambda_{122}^{\prime} / m_{\tilde{f}}^{2}$ predict a too large branching ratio of the neutralino into invisible final states and hence too small values of $\lambda_{112}^{\prime} / m_{\tilde{f}}^{2}$ below $1 \times 10^{-11} \mathrm{GeV}^{-2}$ cannot be probed, regardless of the value of $\lambda_{\text {prod }}^{\prime} / m_{\tilde{f}}^{2}$. This effect becomes more prominent in the results shown in a different parameter plane below.

For scenario $2, \lambda_{\text {prod }}^{\prime}$ does not produce any invisible final state from neutralino decays, see Table II, which is why there is no lower bound on the sensitivity to $\lambda_{\text {dec }}^{\prime} / m_{\tilde{f}}^{2}=\lambda_{112}^{\prime} / m_{\tilde{f}}^{2}$. For this scenario, AL3X is sensitive as long as the product $\lambda_{112}^{\prime} \lambda_{131}^{\prime} / m_{\tilde{f}}^{4}$ is larger than $\approx 2 \times 10^{-18} \mathrm{GeV}^{-4}$.

In Fig. 5, we show the same results in a different parameter plane, i.e., the overall branching ratio of the initial state mesons into visible final states, BR(Meson $\rightarrow$ $\left.\tilde{\chi}_{1}^{0}+X\right) \times \operatorname{BR}\left(\tilde{\chi}_{1}^{0} \rightarrow\right.$ charged final state $)$ vs the decay length, $c \tau$, of the neutralino. Here we also overlay results from other LLP experiments, i.e., MATHUSLA, CODEX-b and FASER, as determined in Ref. [16], as well as SHiP from Ref. [6]. We also compare the impact of integrated luminosity and show the expected sensitivity at AL3X for both $100 / \mathrm{fb}$ and $250 / \mathrm{fb}$. Remember that in scenario 1 , increasing $\lambda_{\text {prod }}^{\prime}$ simultaneously increases $\operatorname{Br}($ Meson $\rightarrow$ $\left.\tilde{\chi}_{1}^{0}+X\right)$ and decreases $c \tau$. For that reason there exists a region in this parameter plane which is theoretically impossible and we marked this as the hashed region in the upper right corner in the left plot of Fig. 5. No such effect exists for scenario 2 .

For scenario 1, visible branching ratios down to $\approx 8 \times 10^{-14}$ can be probed with AL3X while for scenario 2 


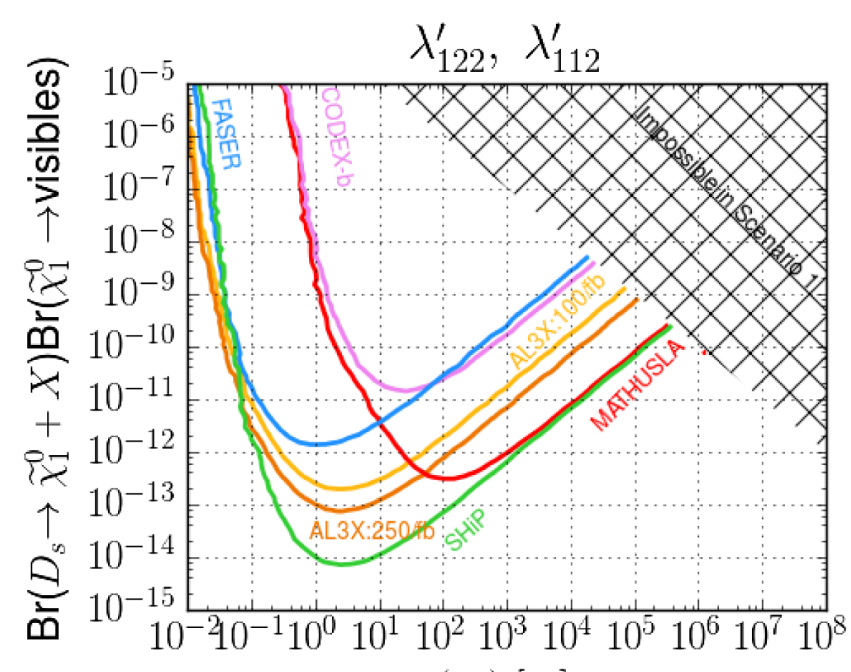

(cr) $[\mathrm{m}]$

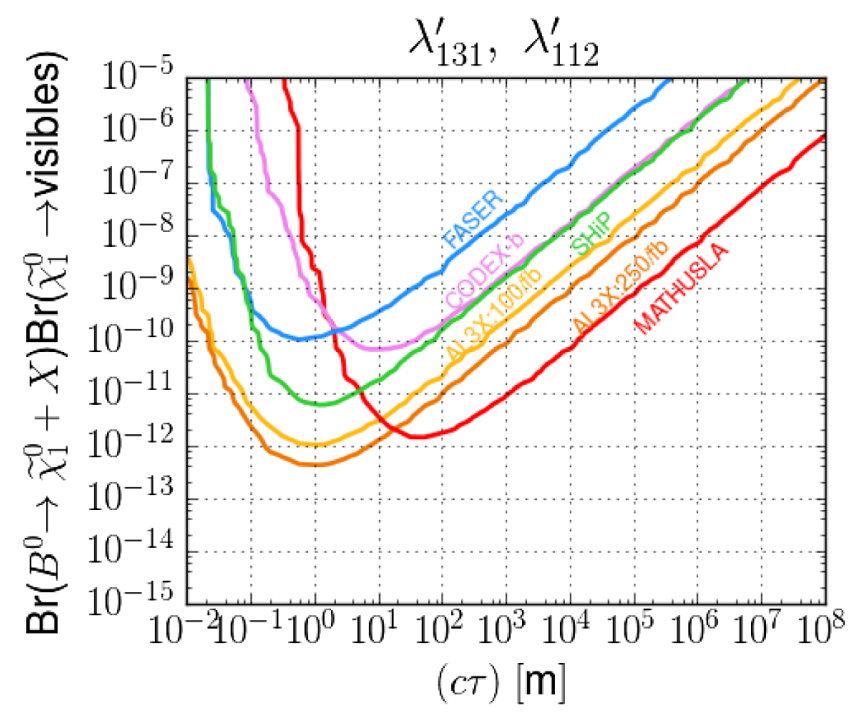

FIG. 5. Model-independent sensitivity estimates for different experiments. We show the sensitivity reach as isocurves of 3 events of visible decays. For the axes, we choose the neutralino's unboosted decay length $c \tau$ and the relevant meson branching ratio times the relevant neutralino branching ratio. For scenario 1, regions with large $c \tau$ and large branching ratio are impossible to construct theoretically.

the sensitivity is about a factor of 5 weaker. The difference can be explained by considering the difference in meson production rates, see Eq. (1), the respective fiducial efficiencies, see Table I, and the effect of invisible decays only present in the first scenario, see Table II.

In comparison with the other experiments, we see that AL3X outperforms both FASER and CODEX-b in both scenarios. As scenario 1 relies on the abundant production of $D$-mesons, SHiP which works at a center-of-mass energy of $\approx 27 \mathrm{GeV}$ is significantly more sensitive. AL3X can only improve on the expected SHiP bound for scenarios with mean decay paths below $0.1 \mathrm{~m}$, due to the proximity of the detector to IP2.
Comparing to the expected sensitivity curves from MATHUSLA, we observe that AL3X can obtain far stronger results for models, with $c \tau$ below roughly $20 \mathrm{~m}$. Again, this can be explained by the different geometry: whilst AL3X is designed with a target-to-detector-distance of about $5 \mathrm{~m}$ close to the IP, MATHUSLA is planned as a surface experiment with a respective distance of more than $140 \mathrm{~m}$.

\section{CONCLUSIONS}

In this work we have investigated the sensitivity of the recently proposed detector AL3X for detecting long-lived fermions in the context of heavy neutral leptons (HNLs), also known as singlet neutrinos, and the lightest neutralino of supersymmetry. For the HNLs study, we present results where solely the mixing between $\nu_{e / \mu}$ and the HNL, $N$, is nonvanishing. For the neutralino case, we consider two production mechanisms: neutralino pair-production from on-shell $Z$-boson decays via the Higgsino component of the neutralinos, and single neutralino production from $D$ - or $B$-meson decays via an RPV $L Q \bar{D}$ coupling. In the study of neutralinos produced from a meson, we take two benchmark scenarios from Refs. [6,16] for illustration of our results. Scenario 1 has the neutralino produced from a $D_{s^{-}}$ meson decay while scenario 2 from a $B^{0}$-meson decay.

We present our HNL results in the mixing angle squared, $\left|V_{\alpha N}\right|^{2}$, vs mass, $m_{N}$, plane, where $\alpha=e, \mu$, cf. Fig. 2. We consider AL3X with $100 /$ fb or $250 /$ fb integrated luminosity, and compare with theoretical projections of other proposed detectors. We find that AL3X reaches smaller mixing angles than both FASER and CODEX-b in its whole mass reach, but is weaker than MATHUSLA by a factor $\sim 3$ for masses below $\sim 4 \mathrm{GeV}$. Compared to SHiP, AL3X is worse in mixing angle reach by one order of magnitude for $m_{N}$ below the $D$-meson threshold, $\sim 2 \mathrm{GeV}$, and is slightly better than SHiP, for larger mass values.

As for detecting neutralinos pair-produced from $Z$-boson decays, we present two plots respectively for $\operatorname{BR}(Z \rightarrow$ $\left.2 \tilde{\chi}_{1}^{0}\right)=10^{-3}$ at the experimental upper limit and for $\operatorname{BR}\left(Z \rightarrow 2 \tilde{\chi}_{1}^{0}\right)=10^{-5}$, switching on a single $L Q \bar{D}$ coupling: $\lambda_{112}^{\prime}$, for the neutralino decay. The plots are shown in the plane $\lambda_{112}^{\prime} / m_{\tilde{f}}^{2}$ vs $m_{\tilde{\chi}_{1}^{0}}$, cf. Fig. 3. We find AL3X, comparable to other detectors, has a mass reach from $\sim 1 \mathrm{GeV}$ up to $\sim m_{Z} / 2$. While MATHUSLA has the strongest reach in $\lambda_{112}^{\prime} / m_{\tilde{f}}^{2}$, AL3X is only slightly worse by a factor $\sim 2$. Novel parameter space, which is orders of magnitude more sensitive than the present experimental limits on $\lambda_{112}^{\prime} / m_{\tilde{f}}^{2}$, can be probed by all of these detectors.

We show two sets of plots for the light neutralinos singly produced from a charm or bottom meson, where two $L Q \bar{D}$ couplings are switched on: $\lambda_{\text {prod }}^{\prime}$ and $\lambda_{\mathrm{dec}}^{\prime}$, responsible for the production and the decay of the lightest neutralino, respectively. In the first set, Fig. 4, shown in the plane $\lambda_{\text {prod }}^{\prime} / m_{\tilde{f}}^{2}$ vs $\lambda_{\text {dec }}^{\prime} / m_{\tilde{f}}^{2}$ for three representative values of $m_{\tilde{\chi}_{1}^{0}}$, 
we find that in scenario $1, \mathrm{AL} 3 \mathrm{X}$ has a reach in $\lambda_{\text {prod } / \mathrm{dec}}^{\prime} / m_{\tilde{f}}^{2}$ beyond existing bounds by roughly an order of magnitude, but weaker than SHiP [6], by approximately a factor of 3. In the other scenario associated with a $B^{0}$-meson, AL3X can go beyond existing limits by almost two orders of magnitude, and is more sensitive than SHiP by about a factor of 5 in both axes. In the second set of plots, Fig. 5, we present results in the plane $\operatorname{Br}\left(\right.$ meson $\left.\rightarrow \tilde{\chi}_{1}^{0}\right) \cdot \operatorname{Br}\left(\tilde{\chi}_{1}^{0} \rightarrow\right.$ visibles $)$ vs $c \tau$, the decay length of $\tilde{\chi}_{1}^{0}$, and compare with other experiments. In scenario 1 , SHiP shows the strongest sensitivity in the product of branching ratios, covering all the sensitive areas of the other proposed detectors, while in scenario 2 AL3X supersedes the whole sensitivity region of SHiP, and complements MATHUSLA in different $c \tau$ regimes.

In summary, we conclude that AL3X can complement or even exceed the other proposed detectors in the parameter space of the different models considered here. It might be interesting to study also other models for this newly proposed detector. Finally, we stress that our sensitivity estimates are based on the assumption of essentially background-free experimental searches. Any unforeseen background could seriously affect these conclusions.

\section{ACKNOWLEDGMENTS}

We thank Torbjörn Sjöstrand, Jordy de Vries and Juan Carlos Helo for useful discussions. H. K. D. and Z.S.W. are supported by the Sino-German DFG Grant No. SFB CRC 110 "Symmetries and the Emergence of Structure in QCD". M. H. acknowledges support by MICINN Grants No. FPA2017-85216-P, No. SEV-20140398 (AEI/FEDER, UE), Red Consolider MultiDark (FPA2017-090566-REDC) and PROMETEOII/2018/165 (Generalitat Valenciana).

Note added.-Recently, an updated sensitivity estimate for SHiP has been published recently in Ref. [58].
[1] R. Essig et al., Working group report: New light weakly coupled particles, in Proceedings, 2013 Community Summer Study on the Future of U.S. Particle Physics: Snowmass on the Mississippi (CSS2013): Minneapolis, MN, USA, 2013 (2013).

[2] S. Alekhin et al., Rep. Prog. Phys. 79, 124201 (2016).

[3] S. Chatrchyan et al. (CMS Collaboration), J. High Energy Phys. 02 (2013) 085.

[4] G. Aad et al. (ATLAS Collaboration), Phys. Rev. Lett. 108, 251801 (2012).

[5] P. Ilten, J. Thaler, M. Williams, and W. Xue, Phys. Rev. D 92, 115017 (2015).

[6] J. de Vries, H. K. Dreiner, and D. Schmeier, Phys. Rev. D 94, 035006 (2016).

[7] D. Curtin et al., arXiv:1806.07396.

[8] V. V. Gligorov, S. Knapen, M. Papucci, and D. J. Robinson, Phys. Rev. D 97, 015023 (2018).

[9] J. L. Feng, I. Galon, F. Kling, and S. Trojanowski, Phys. Rev. D 97, 035001 (2018).

[10] V. V. Gligorov, S. Knapen, B. Nachman, M. Papucci, and D. J. Robinson, Phys. Rev. D 99, 015023 (2019).

[11] D. Choudhury, H. K. Dreiner, P. Richardson, and S. Sarkar, Phys. Rev. D 61, 095009 (2000).

[12] H. K. Dreiner, C. Hanhart, U. Langenfeld, and D. R. Phillips, Phys. Rev. D 68, 055004 (2003).

[13] H. K. Dreiner, O. Kittel, and U. Langenfeld, Phys. Rev. D 74, 115010 (2006).

[14] H. K. Dreiner, S. Heinemeyer, O. Kittel, U. Langenfeld, A. M. Weber, and G. Weiglein, Eur. Phys. J. C 62, 547 (2009).

[15] J. C. Helo, M. Hirsch, and Z. S. Wang, J. High Energy Phys. 07 (2018) 056.
[16] D. Dercks, J. De Vries, H. K. Dreiner, and Z.S. Wang, arXiv:1810.03617.

[17] R. Aaij et al. (LHCb Collaboration), J. High Energy Phys. 03 (2016) 159; 05 (2017) 074(E).

[18] R. Aaij et al. (LHCb Collaboration), Phys. Rev. Lett. 118, 052002 (2017); 119, 169901(E) (2017).

[19] G. Aad et al. (ATLAS Collaboration), Phys. Lett. B 759, 601 (2016).

[20] M. Tanabashi et al. (Particle Data Group), Phys. Rev. D 98, 030001 (2018).

[21] T. Sjostrand, S. Mrenna, and P. Z. Skands, J. High Energy Phys. 05 (2006) 026.

[22] T. Sjöstrand, S. Ask, J. R. Christiansen, R. Corke, N. Desai, P. Ilten, S. Mrenna, S. Prestel, C. O. Rasmussen, and P. Z. Skands, Comput. Phys. Commun. 191, 159 (2015).

[23] A. Atre, T. Han, S. Pascoli, and B. Zhang, J. High Energy Phys. 05 (2009) 030.

[24] W. Porod, Comput. Phys. Commun. 153, 275 (2003).

[25] W. Porod and F. Staub, Comput. Phys. Commun. 183, 2458 (2012).

[26] R. Mohapatra and J. Valle, Phys. Rev. D 34, 1642 (1986).

[27] H. K. Dreiner, H. E. Haber, and S. P. Martin, Phys. Rep. 494, 1 (2010).

[28] F. F. Deppisch, P. S. B. Dev, and A. Pilaftsis, New J. Phys. 17, 075019 (2015).

[29] G. Bernardi et al., Phys. Lett. B 203, 332 (1988).

[30] S. A. Baranov et al., Phys. Lett. B 302, 336 (1993).

[31] F. Bergsma et al. (CHARM Collaboration), Phys. Lett. 166B, 473 (1986).

[32] P. Abreu et al. (DELPHI Collaboration), Z. Phys. C 74, 57 (1997); 75, 580(E) (1997). 
[33] J. P. Chou, D. Curtin, and H. J. Lubatti, Phys. Lett. B 767, 29 (2017).

[34] F. Kling and S. Trojanowski, Phys. Rev. D 97, 095016 (2018).

[35] C. Adams et al. (LBNE Collaboration), arXiv:1307.7335.

[36] K. Bondarenko, A. Boyarsky, D. Gorbunov, and O. Ruchayskiy, J. High Energy Phys. 11 (2018) 032.

[37] M. Drewes, J. Hajer, J. Klaric, and G. Lanfranchi, J. High Energy Phys. 07 (2018) 105.

[38] H. K. Dreiner, Pramana J. Phys. 51, 123 (1998); Adv. Ser. Dir. High Energy Phys. 21, 565 (2010).

[39] R. Barbier et al., Phys. Rep. 420, 1 (2005).

[40] H. P. Nilles, Phys. Rep. 110, 1 (1984).

[41] S. P. Martin, arXiv:hep-ph/9709356; Adv. Ser. Dir. High Energy Phys. 18, 1 (1998).

[42] S. Weinberg, Phys. Rev. D 26, 287 (1982).

[43] H. K. Dreiner, M. Hanussek, and C. Luhn, Phys. Rev. D 86, 055012 (2012).

[44] H. K. Dreiner, C. Luhn, and M. Thormeier, Phys. Rev. D 73, 075007 (2006).

[45] H. K. Dreiner, C. Luhn, H. Murayama, and M. Thormeier, Nucl. Phys. B774, 127 (2007).

[46] F. de Campos, O. J. P Éboli, M. B. Magro, W. Porod, D. Restrepo, M. Hirsch, and J. W. F. Valle, J. High Energy Phys. 05 (2008) 048.
[47] K. Desch, S. Fleischmann, P. Wienemann, H. K. Dreiner, and S. Grab, Phys. Rev. D 83, 015013 (2011).

[48] D. Dercks, H. Dreiner, M. E. Krauss, T. Opferkuch, and A. Reinert, Eur. Phys. J. C 77, 856 (2017).

[49] B. C. Allanach, A. Dedes, and H. K. Dreiner, Phys. Rev. D 60, 075014 (1999).

[50] Y. Kao and T. Takeuchi, arXiv:0910.4980.

[51] H. K. Dreiner, S. Grab, D. Koschade, M. Krämer, B. O'Leary, and U. Langenfeld, Phys. Rev. D 80, 035018 (2009).

[52] F. Domingo, H K. Dreiner, J. S. Kim, M. E. Krauss, V. M. Lozano, and Z. S. Wang, J. High Energy Phys. 02 (2019) 066.

[53] A. Bartl, W. Majerotto, and N. Oshimo, Phys. Lett. B 216, 233 (1989).

[54] C. Patrignani et al. (Particle Data Group), Chin. Phys. C 40, 100001 (2016).

[55] K. Agashe and M. Graesser, Phys. Rev. D 54, 4445 (1996).

[56] B. C. Allanach, A. Dedes, and H. K. Dreiner, Phys. Rev. D 60, 056002 (1999); 86, 039906(E) (2012).

[57] B. C. Allanach, A. Dedes, and H. K. Dreiner, Phys. Rev. D 69, 115002 (2004); 72, 079902(E) (2005).

[58] SHiP Collaboration, arXiv:1811.00930. 